Bond University

Research Repository

\title{
Legacy effect of delayed blood pressure lowering drug treatment in middle-aged adults with mildly elevated blood pressure: systematic review and meta-analysis
}

Ho, Chau L B; Sanders, Sharon; Breslin, Monique; Doust, Jenny; Reid, Christopher M; Davis, Barry R; Simpson, Lara M; Brouwers, Frank P; Nelson, Mark R

Published in:

Journal of Human Hypertension

DOI:

10.1038/s41371-020-0323-7

Licence:

Other

Link to output in Bond University research repository.

Recommended citation(APA):

Ho, C. L. B., Sanders, S., Breslin, M., Doust, J., Reid, C. M., Davis, B. R., Simpson, L. M., Brouwers, F. P., \& Nelson, M. R. (2020). Legacy effect of delayed blood pressure lowering drug treatment in middle-aged adults with mildly elevated blood pressure: systematic review and meta-analysis. Journal of Human Hypertension, 34(4), 261-270. https://doi.org/10.1038/s41371-020-0323-7

\section{General rights}

Copyright and moral rights for the publications made accessible in the public portal are retained by the authors and/or other copyright owners and it is a condition of accessing publications that users recognise and abide by the legal requirements associated with these rights.

For more information, or if you believe that this document breaches copyright, please contact the Bond University research repository coordinator. 
1 Title: Legacy effect of delayed blood pressure lowering drug treatment in

2 middle-aged adults with mildly elevated blood pressure: systematic review and

3 meta-analysis

4

5 Running title: Legacy effect of BP lowering drug treatment in primary prevention

6 Authors: Chau L. B. Ho ${ }^{1,2}$, Sharon Sanders ${ }^{3}$, Monique Breslin ${ }^{1}$, Jenny Doust ${ }^{3}$,

7 Christopher M. Reid ${ }^{2,4}$, Barry R. Davis ${ }^{5}$, Lara M. Simpson ${ }^{5}$, Frank P. Brouwers ${ }^{6}$,

8 Mark R. Nelson ${ }^{1,4}$.

9 Affiliations: ${ }^{1}$ Menzies Institute for Medical Research, University of Tasmania, Hobart,

10 Australia, ${ }^{2}$ School of Public Health, Curtin University, Perth, Australia, ${ }^{3}$ Institute for

11 Evidence-Based Healthcare, Bond University, Gold Coast, Australia, ${ }^{4} \mathrm{CCRE}$

12 Therapeutics, School of Public Health and Preventive Medicine, Monash University,

Melbourne, ${ }^{5}$ University of Texas School of Public Health, Houston, United States, ${ }^{6}$

Department of Cardiology, Haga Teaching Hospital, Postbus 40551, LN The Hague,

The Netherlands

Corresponding author: Dr. Chau L.B. Ho, Menzies Institute for Medical Research,

17 University of Tasmania, Private Bag 23, Hobart TAS 7001, Australia. Tel:

18 +61406656898. Fax: +61362264734. Email: chau.ho@utas.edu.au.

Word count: 3787

Number of tables: 2

Number of figures: 3.

Number of supplementary digital content files: 1 


\section{Abstract}

To investigate if there is evidence for a 'legacy effect' for BP lowering treatment, that is worse health outcomes from not initiating drug treatment at a systolic BP threshold of $140 \mathrm{mmHg}$ in middle-age adults. We systematically reviewed studies comparing the effects of delayed BP treatment (placebo/untreated during the trial or no previous treatment at trial entry) versus early treatment (actively treated during the trial or previous BP treatment at trial entry) on mortality in the short-term (5-year intrial period) and long-term ( $\geq 10$ years in total period). The data were pooled using Peto ORs. A subgroup analysis by 10 -year Framingham risk score was performed. Three studies (ALLHAT, Oslo and PREVEND-IT) involving 4746 participants were included. The results were heavily influenced by the ALLHAT trial. We found no significant difference in all-cause mortality between 'delayed BP' and 'early treatment' in the short-term OR $0.95(95 \% \mathrm{Cl} 0.68-1.32)$ or long-term OR 0.90 $(95 \% \mathrm{Cl} 0.78-1.04)$, with similar results for mortality from cardiovascular disease (CVD). The effects of delayed BP lowering treatment on long-term all-cause and CVD mortality did not vary with baseline risk of CVD. The review showed no clinically adverse 'legacy effect' on mortality or major CVD event from not treating middleaged adults at a systolic BP threshold of $140 \mathrm{mmHg}$ or over. The results were consistent for all CVD risk subgroups. Although these studies are non-randomised post-hoc analyses, they may allay concerns that early treatment of elevated systolic $\mathrm{BP}$ is necessary to prevent CVD events in primary prevention populations.

Key words: legacy effect, blood pressure, long-term, all-cause mortality, CVD mortality, primary prevention, cardiovascular disease 


\section{Introduction}

The effectiveness of blood pressure (BP) lowering drugs to prevent cardiovascular disease (CVD) has been well established in trials of patients with diabetes, the elderly, or those with a systolic BP of $\geq 160 \mathrm{mmHg}$ or over (for example SHEP ${ }^{1}$, Syst-Eur ${ }^{2}$ and HYVET $^{3}$ ). However, the effects of BP lowering pharmacotherapy in middle-aged adults with mildly elevated BP (defined as systolic BP 140-159 mmHg and/or diastolic BP $90-99 \mathrm{mmHg}$ ) are uncertain. A recent systematic review of participants with mildly elevated BP found no statistically significant effect of treatment in this patient group on the incidence of CVD events or mortality (Diao et al ${ }^{4}$ ). However, a similar review by the Blood Pressure Lowering Treatment Trialist's Collaboration (BPLTTC) ${ }^{5}$ observed significant reductions in stroke, CVD and all-cause mortality. Although the BPLTTC review included more trials with a larger number of participants, these trials evaluated both less versus more intensive treatments and the addition of new BP treatment to pre-existing medication and so the comparison was not restricted to active treatment versus placebo/no treatment as in the Diao et al review. In line with the findings in the Diao et al review ${ }^{4}$, most of the placebo trials ${ }^{6-12}$ in which previous treatments were not permitted or were withdrawn, did not show substantial effects of active drug treatment on major CVD events, coronary heart disease $(\mathrm{CHD})$, stroke or all-cause mortality within the trial period.

Concerns have been raised, however, that the effects of delayed treatment may take longer five years to become evident, and that delaying treatment after a patient reaches a SBP threshold of $140 \mathrm{mmHg}$ could result in irreversible pathological damage. Two systematic reviews ${ }^{13,14}$ have been conducted of BP lowering trials with a post-trial follow-up of up to ten years and showed a significantly reduced risk of CVD and all-cause mortality in the participants randomly allocated to active treatment. However, these two reviews included patients with pre-existing CVD. Therefore, the 'legacy effect' of delayed drug treatment in individuals with mildly elevated SBP without cardiovascular disease remains uncertain. As there are no trials that addressed this specific question, the aim of this review is to investigate if there are any adverse 'legacy effects from not initiating drug treatment at a systolic BP threshold of 140 $\mathrm{mmHg}$ in healthy middle-age adults using post-hoc analyses of existing trials with longterm follow up. 


\section{Methods}

\section{Protocol and registration}

The review protocol was published in the Journal of Medical Internet Research ${ }^{15}$ and can be accessed via https://www.researchprotocols.org/2017/9/e177/. The review was registered in PROSPERO International Prospective Register of Systematic Reviews: CRD42017058414

\section{Criteria for considering studies for this review}

The current review included randomised controlled trials (RCTs) with at least 1-year post-trial follow-up. Trials including men and non-pregnant women from 30 to 65 years of age, where at least $80 \%$ of participants had mildly elevated BP (defined as a systolic BP of $140-159 \mathrm{mmHg}$ ) and no history of CVD (myocardial infarction, angina pectoris, coronary bypass surgery, coronary angioplasty, stroke, transient ischaemic attack, carotid endarterectomy, surgery for peripheral vascular disease, intermittent claudication or renal failure (creatinine > 1.5 times the upper limit of normal)) at baseline were eligible. We included studies that used a placebo or untreated control comparator or another active BP lowering treatment where it was possible to determine participants who had previously been taking blood pressure lowering treatment (previous treatment) or no pre-existing treatment (treatment naïve). Where trials included participants different to those of interest (e.g. in secondary prevention populations, in participants with moderately or highly elevated BP or older than 65 years), we attempted to access data from trial investigators and subsequently included only participants meeting our criteria in the analyses. The primary outcome of the review was all-cause mortality, with secondary outcome of CVD mortality and CVD events (defined as fatal and non-fatal stroke, fatal and non-fatal CHD, fatal and non-fatal heart failure).

\section{Data sources and searches}

We searched Medline via Ovid (1946 to Sept 2018), Embase via Ovid (1974 to Sept 2018) and the Cochrane Register of Controlled Trials (CENTRAL) (Sept 2018). We combined text word and MeSH/Emtree terms related to BP lowering drug agents with hypertension terms and follow-up studies. We used the Cochrane Highly Sensitive Search Strategy for identifying randomised trials (sensitivity and precision maximising 2008 revision) in Medline ${ }^{16}$. No language restrictions were applied. The search 
strategies are provided in Table appendix 1. We modified the search strategy from the published protocol ${ }^{15}$ as the planned method of identifying trials and then searching for follow-up studies was considered inadequate to identify potentially eligible RCTs.

We searched reference lists of known systematic reviews on post-trial studies of BP lowering drug treatment (Kostis $2010^{13}$ and Hirakawa 2017 ${ }^{14}$ ) and meta-analyses of trials in middle-aged adults with mildly elevated $\mathrm{BP}^{4}, 5,17,18$. We contacted corresponding authors of relevant papers regarding any further published or unpublished work.

\section{Study selection}

Two reviewers ( $\mathrm{CH}$ and $\mathrm{SS}$ ) independently scanned the results of the title and abstract search and any potentially relevant articles were obtained in full text. Two reviewers then screened the full text of potentially relevant articles against the reviews inclusion criteria. Discrepancies were resolved through discussion with a third reviewer.

\section{Data extraction}

Data extraction were independently performed by two reviewers ( $\mathrm{CH}$ and $\mathrm{SS})$. If any disagreement arose, a third reviewer (JD) was consulted. The extraction form included details of study characteristics, participant characteristics, interventions and settings, outcome data, type of analysis used in the studies and follow-up years.

\section{Assessment of risk of bias in included studies}

Two review authors ( $\mathrm{CH}$ and $\mathrm{SS}$ ) independently assessed risk of bias using the Cochrane Risk of bias in non-randomised and /randomised studies of interventions tools 19, 20. The included ALLHAT study was assessed using the tool for nonrandomised studies as data from the original randomised trial was reanalysed to compare non-randomised groups (treatment naïve vs previous treatment) based on data collected at trial baseline. Risk of bias assessment in both non-randomised ${ }^{21}$ and randomised studies ${ }^{22}$ included consideration of four mutual domains: bias due to deviations from intended interventions, bias due to missing data, bias in measurement of outcomes and bias in selection of the reported. Risk of bias assessment in nonrandomised controlled studies required consideration of three further criteria: bias due to confounding, bias in selection of participants into the study and bias in classification of intervention. For randomised studies, risk of bias assessment also included consideration of bias arising from the randomisation process. For the non-randomised 
studies, each risk of bias domain was assessed as low, moderate, serious or critical risk of bias with a no information response when insufficient data were reported to permit a judgment. For the randomised studies, each risk of bias domain was assessed as low, some concerns and high risk of bias. The domain level judgments provide the basis for an overall risk of bias judgment for each study. An assessment of potential publication bias was not performed due to the small number of included studies.

\section{Data analysis}

We compared outcomes in the short-term (average 5-year in-trial period) and longterm (an overall period of at least 10 years cumulative in- and post-trial period) between 'delayed treatment' and 'early treatment' groups. The 'early treatment' group included who had been previously treated with blood pressure lowering treatment at trial entry and the 'delayed treatment' group included participants who were treatment naïve using individual patient data from the trial. This approach has been used previously by Nelson et al ${ }^{23}$.

Due to the small number of included studies, fixed effect Peto odds ratio (OR) was used to estimate the pooled effects ${ }^{24}$. As recommended ${ }^{25-28}$, we also used other methods to test the robustness of the results in sensitivity analyses. Heterogeneity of treatment effects in different trials was tested by the $\mathrm{I}^{2}$ statistic. Statistical heterogeneity was recorded when the $p$ value of the test of heterogeneity was 0.1 or lower or the $\mathrm{I}^{2}$ value was 0.5 or greater. In a post-hoc analysis of the ALLHAT trial, the effects of 'no previous treatment' versus 'previous treatment' for high BP were estimated using a Cox proportional hazard model. As this analysis was a comparison of non-randomised groups, the two groups were adjusted for an imbalance in baseline characteristics (e.g. age, race, sex, diabetes mellitus, education, body mass index, smoking, aspirin use, randomised group, BP, total cholesterol, serum glucose and creatinine), as per Nelson et al in the ANBP2 study23. The observed (O), expected event (E) and variance (V) in ALLHAT were estimated from adjusted HR as recommended by Tierney et al ${ }^{29}$ and then pooled with the corresponding $\mathrm{O}, \mathrm{E}$ and $\mathrm{V}$ in Oslo and PREVEND-IT. The threshold of a significant effect was set at 0.05.

We conducted a sub-group analysis based on baseline risk of CVD where data were available. We stratified participants by the baseline estimated 10-year Framingham risk score for fatal and non-fatal CVD events using thresholds of lower 
than 20\% (low risk), 20-30\% (moderate risk) and higher than 30\% (high risk) over 10 years ${ }^{30,31}$. We estimated the relative risk for all-cause and CVD mortality in each group and tested for difference between the groups. Data synthesis and analyses were performed in Review Manager $5{ }^{32}$. We extracted data based on intention-to-treat principles.

\section{Sensitivity analysis}

An analysis restricted to placebo/untreated controlled RCTs was performed to investigate the impact of the observational study on the pooled outcomes. Different statistical methods were also used to check the robustness of the results ${ }^{25-28}$.

\section{Results}

\section{Result of the searches}

The database searches identified 6012 records and three articles were identified from other sources (Figure Appendix 1 shows the flowchart of studies). After removal of duplicates 4090 articles were screened. Eighty nine articles were screened in full-text and 3 studies (Oslo, PREVEND-IT and ALLHAT) from 11 articles were included in the review. Aggregate unpublished data from the ALLHAT and individual data of PREVEND-IT trial were provided by the trial investigators.

One trial excluded from the review included participants with mildly elevated diastolic BP (90-115 mmHg): USPHS 197733, 34. Although USPHS did not have a post-trial phase, the trial was followed for up to 10 years. No information on the proportion of participants with mildly elevated systolic BP was reported. Based on the baseline systolic BP $148 \pm 15 \mathrm{mmHg}$, it is likely that less than $80 \%$ of participants had systolic BP less than $160 \mathrm{mmHg}$. The intervention was a combination of a diuretic and rauwolfia serpentine that had limited clinical use in current practice because of the risk of side effects and availability. Thus USPHS was excluded in the current systematic review and meta-analysis.

\section{Characteristics of included studies and risk of bias}

The review included published data from the Oslo trial, unpublished aggregate data from the ALLHAT and individual data from the PREVEND-IT. In the ALLHAT trial, we used data based on whether participants had previously been treated with BP lowering agents or not, that is a comparison on a difference in treatment status at baseline between the two groups rather than a randomised comparison. ALLHAT participants 
were followed for a mean of 4.9 years in the in-trial period and 14 years over the inand post-trial period. As the original ALLHAT trial ${ }^{35}$ reported beneficial effects from BP lowering treatment (e.g. Chlorthalidone 12.5 to $25 \mathrm{mg} / \mathrm{d}$ vs amlodipine 2.5 to 10 $\mathrm{mg} / \mathrm{d}$ vs lisinopril 10 to $40 \mathrm{mg} / \mathrm{d}$ ) within the trial period, the majority of participants from all arms of the trials received active treatment in the post-trial phase, so there is likely to be little cross-over between the early treatment and delayed treatment comparison groups. Although some participants in the Oslo trial may have had a diastolic BP exceeding $110 \mathrm{mmHg}$, nearly $80 \%$ of Oslo participants had systolic BP lower than 160 $\mathrm{mmHg}$, so we included the published data of this trial. Oslo participants were randomised to active treatment (Hydrochlorothiazide $50 \mathrm{mg}$ ) or no active treatment. Oslo reported 10 -year ${ }^{36}$ and 40 -year ${ }^{37}$ follow-up of all-cause mortality and CHD mortality, thus the results of the 40-year study were included in the current review. In PREVEND-IT trial, participants were originally randomised either to active treatment (Fosinopril $20 \mathrm{mg}$ ) or placebo. The mean follow-up period ranged from 3.3-4.4 years for the in-trial phase and 9.4-10.7 years for the overall period.

The baseline risk for participants in ALLHAT was higher than the other two trials as it included participants with elevated BP and at least one other CVD risk factor (e.g. history of type 2 diabetes, current cigarette smoking, high-density lipoprotein cholesterol of less than $0.91 \mathrm{mmol} / \mathrm{L})$. PREVEND-IT included healthy subjects from the general population with persistent microalbuminuria, and the Oslo trial included men with mildly elevated BP (defined as systolic BP 150-179 mmHg and diastolic BP less than $110 \mathrm{mmHg}$ ). More details on the characteristics of the included and excluded studies are provided in Table appendix 2 and 3.

The baseline characteristics of the participants included in the review showed no significant differences between study groups in the PREVEND-IT and Oslo trials (Table 1). ALLHAT participants had a higher proportion of patients with diabetes, and contributed to a higher proportion of participants with early treatment having type 2 DM. Participants with early treatment in the ALLHAT trial were also more likely to be black, female, non-smoker and had higher estimated 10-year CVD risk scores. We adjusted for these imbalances in multivariable models. Noticeably, Oslo included men only and had higher baseline systolic BP than the other two trials.

\section{Risk of bias (Table 2)}


In Table 2, We assessed the ALLHAT data to be at serious risk of bias due to residual confounding as a result of the use of post-hoc non-randomised data from the trial. Although the outcome measurements in the post-trial phase of the PREVEND-IT and Oslo trials were unblinded, the primary outcomes considered in this analysis are generally objective (all-cause and cardiovascular mortality). Thus, the overall risk of bias for the PREVEND-IT and Oslo trials were judged as 'Low risk'. More details on the assessment of the risk of bias in each trial are presented in Table appendix 4.

\section{Short- and long-term all-cause and CVD mortality (Figure 1)}

The analyses on short- and long-term all-cause mortality and short-term CVD mortality included 4746 participants from three trials, with $80 \%$ originating from the ALLHAT trial. As the Oslo trial separately reported aggregate data for $\mathrm{CHD}$ and stroke, these subjects were excluded in the analysis of long-term CVD mortality, leaving 3961 participants in the analysis. There were 301 deaths in total and 102 deaths due to CVD recorded in the in-trial period, and 1871 total deaths and 312 CVD deaths during the post-trial period (Table appendix 5).

In Figure 1, we observed no statistically significant difference in all-cause mortality in either the short- or long-term (short-term OR $0.95,95 \% \mathrm{Cl} 0.68-1.32$; long-term OR $0.90,95 \% \mathrm{Cl} 0.78-1.04$ ) for those with delayed $\mathrm{BP}$ lowering treatment relative to those with earlier treatment. Similarly, no difference was found for CVD mortality (short-term OR 0.90, 95\% Cl 0.51-1.59; long-term OR 0.79, 95\% Cl 0.55-1.14).

\section{CVD events (Figure 1)}

Two trials (Oslo and PREVEND-IT) including 934 participants contributed to the analysis of major CVD events in the short-term, with 69 events recorded in the in-trial phase of the Oslo and PREVEND-IT trials. However, only PREVEND-IT (149 participants, 19 events) recorded long-term outcomes ${ }^{38}$. As provided in Figure 1, we found no statistically significant difference in major CVD events for those with delayed drug treatment in either the short or long-term (short-term OR 1.35, 95\% 0.83-2.21; long-term OR 1.02, 95\% 0.39-2.66).

\section{Subgroup analysis by 10 -year Framingham risk score}

Data were available to stratify participants in ALLHAT and PREVEND-IT into low, moderate and high risk of CVD. More than half of the included participants were in the high risk group, primarily due to the inclusion criteria of the ALLHAT study. The effects of delayed BP lowering drug treatment were consistent among the three groups 
$274 \quad(p=0.46$ and $p=0.79$ for the test of subgroup differences in overall all-cause and CVD 275 mortality respectively) (Figure 2 and Figure 3).

\section{Sensitivity analysis}

277 Using different methods (DerSimonian-Laird between-study variance estimator and Wald-type confidence intervals, DerSimonian-Laird between-study variance estimator and Hartung-Knapp-Sidik-Jonkman adjusted confidence intervals, Paule-Mandel between-study variance estimator and Hartung-Knapp-Sidik-Jonkman confidence intervals) to pool the aggregate data did not change the main findings in all-cause and CVD mortality as presented in Table appendix 6.

An analysis restricted to the data from the randomised trials only (PREVEND-IT and Oslo), were similar to the main analyses, with no statistically significant difference in for short-term all-cause mortality (OR $0.99,95 \% \mathrm{Cl} 0.43-2.27$ ) or long-term all-cause mortality (OR $0.94,95 \% \mathrm{Cl} 0.70-1.28)$ or short- or long-term CVD mortality (short-term OR 1.26, 95\% Cl 0.42 - 3.76; long-term OR 2.23, 95\% Cl 0.23-21.84) (Table appendix 7).

A sensitivity analysis adjusting for baseline differences, showed no substantial difference between the adjusted and crude hazard ratio for any outcome (Table appendix 8).

\section{Discussion}

The present systematic review and meta-analysis of studies with extended post-trial phase showed no statistically significant difference in all-cause and CVD mortality for participants with 'delayed' drug treatment at a systolic BP threshold of $140 \mathrm{mmHg}$ in middle-aged adults even when the follow-up was extended for more than 10 years. Due to the small number of events in the in-trial period, subgroup analyses were performed only for long-term all-cause and CVD mortality. No heterogeneity of 'delayed' treatment effects was found across the low, moderate and high CVD risk subgroups.

Our findings are similar to two earlier systematic reviews in middle-aged adults without previous CVD $^{39}$ and in middle-aged adults both with and without previous CVD $^{17}$. Trials in these reviews had follow-up durations of approximately five years, except for the USPHS study ${ }^{34}$. The USPHS was followed for 7-10 years and did not show any 
(0.09-2.74). Results from USPHS may not be considered relevant to current populations, however, as this trial used rauwolfia, which is no longer recommended treatment. Similar to our short-term results, the SHEP ${ }^{1}$ and Syst-Eur ${ }^{2}$ trials did not record any substantial benefits of 'early' treatment for all-cause or CVD mortality after an in-trial follow-up of five and two years respectively. However, the effects on CVD mortality became statically significant with a HR 0.86 (0.76-0.97) when the SHEP trial was extended to 14 years ${ }^{40}$ and this 'legacy effect' remained significant at the 22-year follow-up ${ }^{41}$. The reduction in mortality in Syst-Eur remained non-statistically significant after a total follow-up of 6 years ${ }^{42}$, indicating that a longer time for follow-up is required to observe significant 'delayed benefits'. The SHEP and Syst-Eur trials had a 'placebo' arm when participants experienced 'placebo' run-in or withdrawal phase. However these trials were aimed at the elderly with much higher systolic BP values of 160 $\mathrm{mmHg}$ or over compared to the participants considered in our review. HOPE-3 trial in intermediate risk participants also observed no statistically significant difference between the effect of an active treatment and placebo in all-cause or CVD mortality and major CVD event after 5.6 years of follow-up.

Benefits of 'active treatment' or harms of 'no treatment' may require longer than ten years to become evident, particularly for mortality outcomes in middle-aged adults with mildly elevated BP who are at low CVD risk. This is the group that where treatment with blood pressure lowering medication is not clearly of benefit. We have attempted in this review to determine if treatment can safely be delayed in this treatment group. In this review, the average Framingham risk score was $>20 \%$, and so is higher than the low risk patients we would consider where treatment could be delayed. Even in this review, however, no clear evidence of early treatment was observed. The included ALLHAT and Oslo trial ${ }^{37}$ were extended to 14 and 40 years respectively, with no substantial 'legacy effect' on all-cause or CVD mortality of delayed treatment observed, and we observed consistent results across the low, moderate and high CVD risk subgroups.

\section{Strengths and limitation}

This is the first study to systematically review the medical evidence to determine if delaying BP lowering treatment for middle-aged adults with a systolic BP between 140 
and $159 \mathrm{mmHg}$ results in an increase in all-cause or cardiovascular mortality in the short or long term.

In spite of vigorous efforts in accessing individual data to identify eligible participants, only three trials with 4746 participants could be included in the current review. Given the much larger size of ALLHAT trial, the overall results were heavily influenced by the results of this trial. In the ALLHAT trial, information on how long before the start of the trial participants had been on BP lowering treatment was not collected and even if it was, we could not truly know how long someone was hypertensive before it was noted. However, in sensitivity analyses on short- and long-term all-cause mortality, the results of analyses excluding the ALLHAT trial were generally consistent with the overall results.

This review did not examine CHD and stroke mortality separately. Given the small number of studies and the potential for CHD and stroke to be affected by different classes of BP lowering medication ${ }^{43,44}$, we were only able to assess overall and total CVD mortality.

The three included trials lacked BP lowering drug treatment information in the posttrial phase except that an equal percentage of participants receiving drug therapy were reported in PREVEND-IT and Oslo trial. Given the 'positive' findings of the original ALLHAT trial, we believe it is likely that a substantial proportion of both arms of the trial would have used BP lowering therapy after the trial period.

We used the Peto method for meta-analysis because of the small number of included studies. While it is true that the Peto method is open to bias when including studies with imbalance in the comparison groups, this only becomes apparent in combination with a large treatment effect ${ }^{24}$. Also, sensitivity analyses using different statistical methods provided similar pooled effects (Appendix 6).

One of the barriers to adopting the absolute risk approach for decisions regarding BP lowering treatment is the concern that early treatment of mildly elevated BP is necessary to prevent pathological changes that result in CVD events. Our systematic review and meta-analysis showed no clinically adverse 'legacy effect' on mortality outcomes of not treating middle-aged adults at a systolic BP between 140 and 159 $\mathrm{mmHg}$. This study contributes to an area of major concern raised by many clinicians 
368 that early treatment of mildly elevated systolic BP is necessary to prevent CVD events

369 in primary prevention population.

\section{Acknowledgement}

371 The researchers gratefully acknowledge the RACGP Foundation and Therapeutic

372 Guidelines Ltd for their support of this project. We thank Dr Toshiaki Ohkuma and

373 Prof John Chalmers for their kind support on the enquiries related to ADVANCE and

374 ADVANCE-ON study. We thank Mark Jones (Biostatistician, Centre for Research in

375 Evidence Based Practice, Bond University) for his advice on statistical issues. No

376 other funding was received from National Institutes of Health (NIH); Wellcome Trust;

377 Howard Hughes Medical Institute (HHMI); or other granting bodies.

\section{Conflicts of Interest and Source of Funding}

379 C.L.B. Ho is a Ph.D. candidate at Menzies Institute for Medical Research, she has 380 received a Ph.D. scholarship from Merle Weaver Postgraduate Scholarship. M.R.N 381 has served on advisory boards for Sanofi and Bayer in the last 3 years. For the 382 remaining authors none were declared. 


\section{References}

1. SHEP Cooperative Research Group. Prevention of stroke by antihypertensive drug treatment in older persons with isolated systolic hypertension. Final results of the Systolic Hypertension in the Elderly Program (SHEP). Jama 1991;265(24):325564.

2. Staessen Jan A, Fagard Robert, Thijs Lutgarde, Celis Hilde, Arabidze Guramy G, Birkenhäger Willem $\mathrm{H}$, et al. Randomised double-blind comparison of placebo and active treatment for older patients with isolated systolic hypertension. The Lancet 1997;350(9080):757-64.

3. Beckett Nigel S., Peters Ruth, Fletcher Astrid E., Staessen Jan A., Liu Lisheng, Dumitrascu Dan, et al. Treatment of Hypertension in Patients 80 Years of Age or Older. New England Journal of Medicine 2008;358(18):1887-98.

4. Diao D, Wright JM, Cundiff DK, Gueyffier F. Pharmacotherapy for mild hypertension. Cochrane Database of Systematic Reviews 2012;8:CD006742.

5. Sundström Johan, Arima Hisatomi, Jackson Rod, Turnbull Fiona, Rahimi Kazem, Chalmers John, et al. Effects of blood pressure reduction in mild hypertension: a systematic review and meta-analysis. Annals of internal medicine 2015;162(3):18491.

6. DREAM Trial Investigators. Effect of ramipril on the incidence of diabetes. New England Journal of Medicine 2006;355(15):1551-62.

7. THE AUSTRALIAN THERAPEUTIC TRIAL IN MILD HYPERTENSION. The Lancet 1980;315(8181):1261-7.

8. Ho Chau Le Bao, Breslin Monique, Doust Jenny, Reid Christopher M, Nelson Mark R. Effectiveness of blood pressure-lowering drug treatment by levels of absolute risk: post hoc analysis of the Australian National Blood Pressure Study. BMJ open 2018;8(3):e017723.

9. NAVIGATOR Study Group. Effect of valsartan on the incidence of diabetes and cardiovascular events. New England Journal of Medicine 2010;362(16):1477-90. 10. Helgeland Anders. Treatment of mild hypertension: a five year controlled drug trial: the Oslo study. The American journal of medicine 1980;69(5):725-32. 11. Asselbergs Folkert W, Diercks Gilles FH, Hillege Hans L, van Boven Ad J, Janssen Wilbert MT, Voors Adriaan A, et al. Effects of fosinopril and pravastatin on 
cardiovascular events in subjects with microalbuminuria. Circulation 2004;110(18):2809-16.

12. Neaton James D, Grimm Richard H, Prineas Ronald J, Stamler Jeremiah, Grandits Greg A, Elmer Patricia J, et al. Treatment of mild hypertension study: final results. Jama 1993;270(6):713-24.

13. Kostis W. J., Thijs L., Richart T., Kostis J. B., Staessen J. A. Persistence of mortality reduction after the end of randomized therapy in clinical trials of blood pressure-lowering medications. Hypertension 2010;56(6):1060-8.

14. Hirakawa Yoichiro, Arima Hisatomi, Rodgers Anthony, Woodward Mark, Chalmers John. Cumulative in-trial and post-trial effects of blood pressure and lipid lowering: systematic review and meta-analysis. Journal of hypertension 2017;35(5):905-13.

15. Ho Chau Le Bao, Sanders Sharon, Doust Jenny, Breslin Monique, Reid Christopher M, Nelson Mark Raymond. Legacy Effect of Delayed Blood PressureLowering Pharmacotherapy in Middle-Aged Individuals Stratified by Absolute Cardiovascular Disease Risk: Protocol for a Systematic Review. JMIR research protocols 2017;6(9).

16. Lefebvre C, Manheimer E, Glanville J. Chapter 6.4. 11.1 The Cochrane Highly Sensitive Search Strategies for identifying randomized trials in MEDLINE. Cochrane Handbook for Systematic Reviews of Interventions Version;5(0).

17. Musini Vijaya M, Gueyffier Francois, Puil Lorri, Salzwedel Douglas M, Wright James M. Pharmacotherapy for hypertension in adults aged 18 to 59 years. Cochrane Database of Systematic Reviews 2017(8).

18. Hoes Arno W, Grobbee Diederick E, Lubsen Jacobus. Does drug treatment improve survival? Reconciling the trials in mild-to-moderate hypertension. 1995. 19. Higgins Julian P T, Altman Douglas G, Gøtzsche Peter C, Jüni Peter, Moher David, Oxman Andrew D, et al. The Cochrane Collaboration's tool for assessing risk of bias in randomised trials; 2011.

20. Sterne Jonathan AC, Hernán Miguel A, Reeves Barnaby C, Savović Jelena, Berkman Nancy D, Viswanathan Meera, et al. ROBINS-I: a tool for assessing risk of bias in non-randomised studies of interventions. Bmj 2016;355:i4919.

21. Sterne JAC, Higgins JPT, Elbers RG, Reeves BC and the development group for ROBINS-I. Risk Of Bias In Non-randomized Studies of Interventions (ROBINS-I): 
detailed guidance, updated 12 October 2016; 201620 April 2019]; Available from: http://www.riskofbias.info

22. Higgins JPT, Sterne JAC, Savović J, Page MJ, Hróbjartsson A, Boutron I, et al. A revised tool for assessing risk of bias in randomized trials In: Chandler $\mathrm{J}$, McKenzie J, Boutron I, Welch V (editors). Cochrane Methods. Cochrane Database of Systematic Reviews 2016( 10 (Suppl 1)).

23. Nelson Mark R. a, Chowdhury Enayet K. b, Doust Jenny c, Reid Christopher M. b d, Wing Lindon M. H. e. Ten-year legacy effects of baseline blood pressure 'treatment naivety' in the Second Australian National Blood Pressure study. Journal of Hypertension.

24. Brockhaus A Catharina, Bender Ralf, Skipka Guido. The Peto odds ratio viewed as a new effect measure. Stat Med 2014;33(28):4861-74.

25. Bradburn Michael J, Deeks Jonathan J, Berlin Jesse A, Russell Localio A. Much ado about nothing: a comparison of the performance of meta-analytical methods with rare events. Stat Med 2007;26(1):53-77.

26. IntHout Joanna, loannidis John PA, Borm George F. The Hartung-KnappSidik-Jonkman method for random effects meta-analysis is straightforward and considerably outperforms the standard DerSimonian-Laird method. BMC Med Res Methodol 2014;14(1):25.

27. Efthimiou Orestis. Practical guide to the meta-analysis of rare events.

Evidence-based mental health 2018;21(2):72-6.

28. Kuss $O$. Statistical methods for meta-analyses including information from studies without any events-add nothing to nothing and succeed nevertheless. Stat Med 2015;34(7):1097-116.

29. Tierney Jayne F, Stewart Lesley A, Ghersi Davina, Burdett Sarah, Sydes Matthew R. Practical methods for incorporating summary time-to-event data into meta-analysis. Trials 2007;8(1):16.

30. National Vascular Disease Prevention Alliance. Guidelines for the management of absolute cardiovascular disease risk. 2012.

31. Nerenberg Kara A., Zarnke Kelly B., Leung Alexander A., Dasgupta Kaberi, Butalia Sonia, McBrien Kerry, et al. Hypertension Canada's 2018 Guidelines for Diagnosis, Risk Assessment, Prevention, and Treatment of Hypertension in Adults and Children. Can J Cardiol 2018;34(5):506-25. 
481

482

483

484

485

486

487

488

489

490

491

492

493

494

495

496

497

498

499

500

501

502

503

504

505

506

507

508

509

510

511

512

32. The Cochrane Collaboration. Review Manager (RevMan) [Computer program], Version 5.3. Copenhagen: The Nordic Cochrane Centre; 2014.

33. United States Public Health Hospitals Cooperative Study Group. Morbidity and mortality in mild essential hypertension. Circ Res 1972;30(31):110-21.

34. Smith W McFate. Treatment of mild hypertension: results of a ten-year intervention trial. Circ Res 1977;40(5 Suppl 1):198-105.

35. The Allhat Officers and Coordinators for the Allhat Collaborative Research Group. Major outcomes in high-risk hypertensive patients randomized to angiotensin-converting enzyme inhibitor or calcium channel blocker vs diuretic: The antihypertensive and lipid-lowering treatment to prevent heart attack trial (allhat). JAMA 2002;288(23):2981-97.

36. Leren Paul, Helgeland Anders. Coronary heart disease and treatment of hypertension some Oslo study data. The American journal of medicine 1986;80(2):36.

37. Holme I, Kjeldsen Se. Long-term survival in the randomized trial of drug treatment in mild to moderate hypertension of the Oslo study 1972-3. Eur J Intern Med [serial online] 2015;26(2):123-6. Available from:

http://onlinelibrary.wiley.com/o/cochrane/clcentral/articles/814/CN$\underline{01051814 / \text { frame.html }}$

https://ac-els-cdn-com.ezproxy.utas.edu.au/S0953620515000321/1-s2.0S0953620515000321-main.pdf? tid=bb06ea32-cd15-11e7-8a97$\underline{00000 a a c b 362 \& a c d n a t=1511088068 \text { 479981dc9deb895a21d5181b3e5dac72. }}$

38. Brouwers Frank P, Asselbergs Folkert W, Hillege Hans L, de Boer Rudolf A, Gansevoort Ron T, van Veldhuisen Dirk J, et al. Long-term effects of fosinopril and pravastatin on cardiovascular events in subjects with microalbuminuria: ten years of follow-up of Prevention of Renal and Vascular End-stage Disease Intervention Trial (PREVEND IT). American heart journal 2011;161(6):1171-8.

39. Gueyffier F, Froment A, Gouton M. New meta-analysis of treatment trials of hypertension: improving the estimate of therapeutic benefit. Journal of human hypertension 1996;10(1):1-8.

40. Patel Alpesh B, Kostis John B, Wilson Alan C, Shea Michael L, Pressel Sara L, Davis Barry R. Long-term fatal outcomes in subjects with stroke or transient 
513 ischemic attack: fourteen-year follow-up of the systolic hypertension in the elderly

514 program. Stroke 2008;39(4):1084-9.

515 41. Kostis John B, Cabrera Javier, Cheng Jerry Q, Cosgrove Nora M, Deng

516 Yingzi, Pressel Sara L, et al. Association between chlorthalidone treatment of

517 systolic hypertension and long-term survival. JAMA 2011;306(23):2588-93.

518 42. Staessen Jan A, Thijs Lutgarde, Fagard Robert, Celis Hilde, Birkenhäger

519 Willem H, Bulpitt Christopher J, et al. Effects of immediate versus delayed

520 antihypertensive therapy on outcome in the Systolic Hypertension in Europe Trial.

521 Journal of hypertension 2004;22(4):847-57.

522 43. Ettehad Dena, Emdin Connor A, Kiran Amit, Anderson Simon G, Callender

523 Thomas, Emberson Jonathan, et al. Blood pressure lowering for prevention of

524 cardiovascular disease and death: a systematic review and meta-analysis. The

525 Lancet 2016;387(10022):957-67.

526 44. Piepoli Massimo F., Hoes Arno W., Agewall Stefan, Albus Christian, Brotons

527 Carlos, Catapano Alberico L., et al. 2016 European Guidelines on cardiovascular

528 disease prevention in clinical practiceThe Sixth Joint Task Force of the European

529 Society of Cardiology and Other Societies on Cardiovascular Disease Prevention in

530 Clinical Practice (constituted by representatives of 10 societies and by invited

531 experts)Developed with the special contribution of the European Association for

532 Cardiovascular Prevention \&amp; Rehabilitation (EACPR). European Heart Journal $533 \quad 2016 ; 37(29): 2315-81$. 
535 Figure 1. Forest plot for outcomes during the in-trial and overall follow-up.

$\mathrm{Cl}$ : Confidence interval, ALLHAT: Antihypertensive and Lipid-Lowering Treatment to Prevent Heart Attack Trial Lipid-Lowering Trial, PREVEND-IT: Prevention of Renal and Vascular Endstage Disease Intervention Trial.

Figure 2. Forest plot for overall all-cause mortality in subgroup by 10 -year 540 Framingham risk score.

Cl: Confidence interval, ALLHAT: Antihypertensive and Lipid-Lowering Treatment to Prevent Heart Attack Trial Lipid-Lowering Trial, PREVEND-IT: Prevention of Renal and Vascular Endstage Disease Intervention Trial.

544 Figure 3. Forest plot for overall CVD mortality in subgroup by 10-year 545 Framingham risk score.

546 Cl: Confidence interval, ALLHAT: Antihypertensive and Lipid-Lowering Treatment to Prevent 547 Heart Attack Trial Lipid-Lowering Trial, PREVEND-IT: Prevention of Renal and Vascular 548 Endstage Disease Intervention Trial. 


\section{Table 1.Baseline characteristics of included participants}

\begin{tabular}{|c|c|c|c|c|c|c|}
\hline \multirow{2}{*}{ Characteristics } & \multicolumn{3}{|c|}{ Delayed } & \multicolumn{3}{|c|}{ Early } \\
\hline & ALLHAT & PREVEND-IT & Oslo & ALLHAT & PREVEND-IT & Oslo \\
\hline $\begin{array}{l}\text { Number of } \\
\text { observations, } \mathrm{n}\end{array}$ & 509 & 70 & 379 & 3303 & 79 & 406 \\
\hline $\begin{array}{l}\text { Age (mean } \pm S D \text {, } \\
\text { years) }\end{array}$ & $59.5 \pm 2.9$ & $52.3 \pm 8.0$ & $45.2 \pm 2.8$ & $59.5 \pm 2.9$ & $50.3 \pm 8.2$ & $45.3 \pm 2.9$ \\
\hline Black, \% & $34.6 *$ & 0 & NA & 43.6 & 1.3 & NA \\
\hline Male, \% & $52.8^{*}$ & 64.3 & 100 & 46.3 & 65.8 & 100 \\
\hline $\begin{array}{l}\text { Current Smoker, } \\
\%\end{array}$ & $43.8^{*}$ & 32.9 & 42.5 & 34.6 & 34.2 & 40.9 \\
\hline $\begin{array}{l}\mathrm{BMI}(\text { mean } \pm \mathrm{SD} \\
\left.\mathrm{kg} / \mathrm{m}^{2}\right)^{+}\end{array}$ & $\begin{array}{l}29.9 \pm \\
5.9 *\end{array}$ & $28.1 \pm 4.2$ & NA & $31.3 \pm 7.1$ & $27.7 \pm 4.7$ & NA \\
\hline Diabetes $^{\dagger}(\%)$ & $41.7 *$ & 2.9 & 0 & 51.1 & 2.5 & 0 \\
\hline $\begin{array}{l}\text { SBPs (mean } \pm \\
\text { SD, } m m H g):\end{array}$ & $147 \pm 7^{*}$ & $147 \pm 6$ & $155 \pm 8$ & $146 \pm 8$ & $148 \pm 6$ & $156 \pm 7$ \\
\hline $\begin{array}{l}\text { DBPs (mean } \pm \\
\text { SD, } m m H g):\end{array}$ & $88 \pm 7^{*}$ & $84 \pm 8$ & $96 \pm 7$ & $87 \pm 7$ & $85 \pm 7$ & $97 \pm 7$ \\
\hline $\begin{array}{l}\text { Fasting Serum } \\
\text { Glucose }^{+} \\
\text {(mmol/L) }\end{array}$ & $7.2+3.5 *$ & $5.3 \pm 1.4$ & $6.0 \pm 0.6$ & $7.6+3.8$ & $5.3 \pm 1.8$ & $6.0 \pm 0.6$ \\
\hline $\begin{array}{l}\text { Total cholesterol } \\
\text { (mmol/L) }\end{array}$ & $5.6 \pm 1.1$ & $6.1 \pm 1.1$ & $7.1 \pm 1.2$ & $5.7 \pm 1.2$ & $6.1 \pm 0.9$ & $7.1 \pm 1.2$ \\
\hline $\mathrm{HDL}-\mathrm{c}^{\dagger}(\mathrm{mmol} / \mathrm{L})$ & $1.2 \pm 0.4$ & $1.0 \pm 0.3$ & NA & $1.2+0.4$ & $1.0 \pm 0.3$ & NA \\
\hline $\begin{array}{l}\text { Serum } \\
\text { Creatinine }^{\dagger} \\
\text { (umol/L) }\end{array}$ & $\begin{array}{l}82.2 \pm \\
27.4\end{array}$ & $82.4 \pm 14.0$ & $96.9 \pm 13.7$ & $\begin{array}{l}84.0 \pm \\
27.4\end{array}$ & $84.8 \pm 14.5$ & $97.2 \pm 14.0$ \\
\hline $\begin{array}{l}\text { 10-year FRS, } \\
\text { mean (SD) }\end{array}$ & $\begin{array}{l}27.7 \pm \\
12.8^{*}\end{array}$ & $20 \pm 12$ & NA & $\begin{array}{l}34.2 \pm \\
15.5\end{array}$ & $21 \pm 16$ & NA \\
\hline
\end{tabular}

$552 *$ : $p<0.05$ for the comparison between the delayed and early treatment groups. ALLHAT: Antihypertensive and Lipid-Lowering Treatment to Prevent Heart Attack Trial Lipid-Lowering Trial, PREVEND-IT: Prevention of Renal and Vascular Endstage Disease Intervention Trial. NA: not available. SBP: Systolic Blood Pressure, DBP: Diastolic Blood Pressure, BMI: Body Mass Index, HDL: High Density 


\section{Table 2 Risk of bias}

\begin{tabular}{|c|c|c|c|c|c|c|c|c|c|}
\hline \multirow[t]{2}{*}{ Trial } & \multicolumn{8}{|c|}{ Risk of bias domain } & \multirow[b]{2}{*}{ Overall } \\
\hline & $\begin{array}{l}\text { Confou } \\
\text { nding }\end{array}$ & $\begin{array}{l}\text { Selecti } \\
\text { on of } \\
\text { particip } \\
\text { ants } \\
\text { into the } \\
\text { study }\end{array}$ & $\begin{array}{l}\text { Classif } \\
\text { ication } \\
\text { of } \\
\text { interve } \\
\text { ntions }\end{array}$ & $\begin{array}{l}\text { Randomi } \\
\text { sation } \\
\text { process }\end{array}$ & $\begin{array}{l}\text { Deviatio } \\
\text { ns from } \\
\text { intende } \\
d \\
\text { interven } \\
\text { tions }\end{array}$ & $\begin{array}{l}\text { Miss } \\
\text { ing } \\
\text { data }\end{array}$ & $\begin{array}{l}\text { Measure } \\
\text { ment of } \\
\text { outcome } \\
\mathrm{s}\end{array}$ & $\begin{array}{l}\text { Selec } \\
\text { tion of } \\
\text { the } \\
\text { report } \\
\text { ed } \\
\text { result }\end{array}$ & \\
\hline ALLHAT & Serious & Low & $\begin{array}{l}\text { Moder } \\
\text { ate }\end{array}$ & NA & $\mathrm{NI}$ & $\mathrm{NI}$ & Low & NA & Serious \\
\hline $\begin{array}{l}\text { PREVEN } \\
\text { D-IT }\end{array}$ & NA & NA & NA & Low & Low & Low & Low & Low & Low \\
\hline Oslo & $\mathrm{NA}$ & NA & NA & Low & Low & Low & Low & Low & Low \\
\hline
\end{tabular}

559 NA - not applicable, NI: No Information. ALLHAT: Antihypertensive and Lipid-Lowering Treatment to

560 Prevent Heart Attack Trial Lipid-Lowering Trial, PREVEND-IT: Prevention of Renal and Vascular

561 Endstage Disease Intervention Trial 